\title{
Leaf Classification based on GLCM Texture and SVM
}

\author{
Naveen M. \\ Scientist, HPC, UPE \\ University of Mysore
}

\author{
Vidyashankara M. S. \\ University of Mysore \\ Pavithra B S \\ IIHR, Bangalore
}

\author{
G. Hemantha Kumar \\ Vice Chancellor
}

\begin{abstract}
This paper involves classification of leaves using GLCM (Gray Level Co-occurrence matrix) texture and SVM (Support Vector Machines). GLCM is used for extracting texture feature of leaves. Creating a plant database for quick and efficient classification and recognition is an important step for their conservation. This approach would help to extract useful features of leaf and improve the accuracy of leaf classification. The standard leaf images are subjected to preprocessing. Feature values are extracted from pre-processed image and they are trained and classified. Standard data sets are used for enhancing the properties of the image.
\end{abstract}

\section{Keywords}

Classification, Extraction, SVM, GLCM

\section{INTRODUCTION}

Plants are the essential natural sources of the earth. Plants play an important role in human life by providing shelter and by maintaining a healthy breathable environment. Creating a plant database for quick and efficient classification and recognition is an important step for their conservation. People recognize a specific plant type by characteristics of its leaf like shape, area, color and texture etc. Environment and the climate are largely dependent on plants. Some plants are also grown for production of bio fuels which are less toxic when compared to other toxic gases. . Natural resource managers, especially those interested in grazing and wildlife management must be able to evaluate the presence or absence of many plant species. It involves innovative method of plant recognition system using digital images of plant leaves. Due to global warming and other factors like lack of awareness of plant knowledge, the plant categories are becoming rare and many plants are about to extinct. Because of this, there is a need for easy and efficient recognition and classification of plant by their category. Plants are mostly identified by taxonomists and the process is time consuming. Some of the plants species helps in identifying them are root, stem, leaf, flower, seed and fruit. Leaf is one of the important feature for recognizing a plant due to its availability almost throughout the year. The main aim of this project is to concentrate on the plant classification based on the texture of the leaf. The texture is the interesting area of research for plant leaf classification which includes advanced techniques.

\section{LITERATURE REVIEW}

According to Rashad, et al.,[1] introduced a novel approach for classification of plants which was based on the characterization of texture properties. They have utilized a combined classifier learning vector quantization along with the radial basis function. The proposed systems ability to classify and recognize a plant from a small part of the leaf is its advantageous thing. Without the need to depend either on the shape of the full leaf or its color features, one can classify a plant having only a portion available that is in itself enough as the proposed system requires only textural features. This system can be useful for the researchers of Botany who need to identify damaged plants, as it can now be done from a small available part. This system is mostly applicable as the combined classifier method produced high performance far superior to other tested methods as its correct recognition rate was $98.7 \%$ which has been revealed in the result. According to Kadir, et al., [2] proposed a method that incorporates shape, vein, color, and texture features. They have used probabilistic neural networks (PNN) as a classifier for the plant leaf classification. Commonly several methods are there for plant leaf classification but none of them have captured color information, because color was not recognized as an important aspect to the identification. Sumathi, et al., [3] proposed a feature fusion technique using the Gabor filter in the frequency domain and fusing the obtained features with edge based feature extraction. The extracted features were trained using 10 fold cross validation and tested with CART and RBF classifiers to measure its accuracy. In the research paper contributed by Beghin, et al., [4] introduced an approach that combines relatively simple methods which used shape and texture features. The shape-based method extracts the contour signature from every leaf and then calculates the dissimilarities between them. The orientations of edge gradients are used to analyze the macro-texture of the leaf. The results of these methods are then combined with the help of incremental classification algorithm which provides $81.1 \%$ accuracy. The research document introduced by Shayan Hati, et al., [7] includes an article that describes how Artificial Neural Network is used to identify plant by inputting leaf image. Compared to earlier approaches, new input features and image processing approach that matter in efficient classification in Artificial Neural Network have been introduced. Image processing techniques are used to extract leaf shape features such as aspect ratio, width ratio, apex angle, apex ratio, base angle, centroid deviation ratio, moment ratio and circularity. These extracted features are used as inputs to neural network for classifying the plants. Under the current research, 534 leaves of 20 kinds of plants were collected. Out of these, 400 leaves were trained. The 134 testing samples were recognized with 92\% accuracy; even without considering types of leaf margins, vein and removal of the petiole. Software has also been developed to identify leaf automatically except two mouse clicks by the user.

\section{PROPOSED METHODOLOGY}

The proposed project involves classification of different species of leaves based on their texture. The leaf images are first subjected to preprocessing which involves resizing the image to standard size then converting the image to grayscale image. In training the leaf images are trained by applying GLCM methodology next the extracted feature values are stored in the database The texture features are extracted using GLCM (Gray level co-occurrence matrix).GLCM creates a gray-level co-occurrence matrix (GLCM) from particular image. It calculates how often a pixel with gray-level (grayscale intensity) value $\mathrm{i}$ occurs horizontally adjacent to a 
pixel with the value $\mathrm{j}$. The GLCM involves several properties and parameters for extracting the texture feature.SVM (support vector machines) are used for classification of different species of leaves.SVM is one of the most useful techniques in classification problems. It is a binary classification technique.

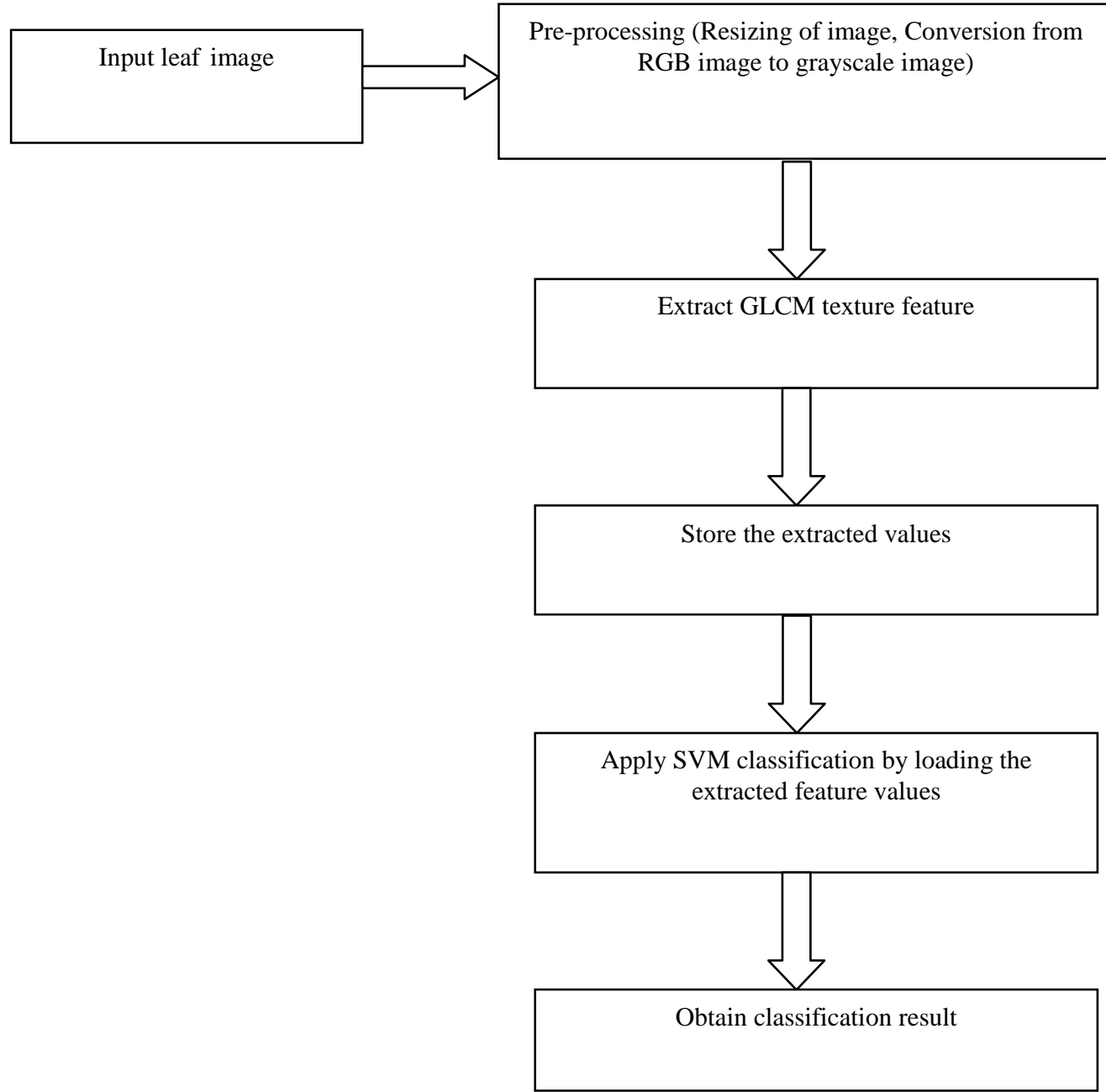

Fig 1: Proposed System

\section{A. GLCM}

The texture features are extracted using GLCM (Gray level co-occurrence matrix).GLCM creates a gray-level cooccurrence matrix (GLCM) from particular image. It calculates how often a pixel with gray-level (grayscale intensity) value i occurs horizontally adjacent to a pixel with the value $\mathrm{j}$. The GLCM involves several properties and parameters for extracting the texture features.

\section{Properties of GLCM}

1.Contrast: Returns a measure of the intensity contrast between a pixel and its neighbor over the whole image.

$$
\text { Con }=\sum_{i=0}^{N-1} \sum_{j=0}^{N-1}(i-j)^{2} M(i, j)
$$

\section{Correlation}

Returns a measure of how correlated a pixel is to its neighbor over the whole image.

$C=\frac{\sum_{i=0}^{N-1} \sum_{j=0}^{N-1} i j M(i, j)-I_{2}^{2}}{I_{3}}$

\section{Energy}

Returns the sum of squared elements in the GLCM.

$$
E=\sqrt{\sum_{i=0}^{N-1} \sum_{j=0}^{N-1} M^{2}(i, j)}
$$

4. Homogeneity

Returns a value that measures the closeness of the distribution of elements in the GLCM to the GLCM diagonal.

$$
H=\sum_{i=0}^{N-1} \sum_{j=0}^{N-1} \frac{M(i, j)}{1+|i-j|}
$$

\section{B. $S V M$}

Support vector machines (SVMs) are used to handle a classical two class pattern recognition problem. It is a supervised machine learning algorithm which can be used for both classification and regression challenges. However itis mostly used in classification problems. Traditional SVM returns a binary value, the class of the object. We are interested in the following two classes: the dissimilarities between images of the same species, and dissimilarities between images of different species. You can use a support vector machine (SVM) when your data has exactly two classes. An SVM classifies data by finding the best hyper plane that separates all data points of one class from those of the other class. The best hyper plane for an SVM means the one with the largest margin between the two classes. Margin 
means the maximal width of the slab parallel to the hyper plane that has no interior data points. An SVM model is a representation of the examples as points in space, mapped so that the examples of the separate categories are divided by a clear gap that is as wide as possible. New examples are then mapped into that same space and predicted to belong to a category based on which side of the gap they fall.

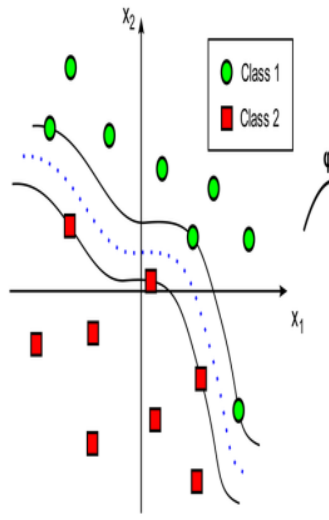

(a)

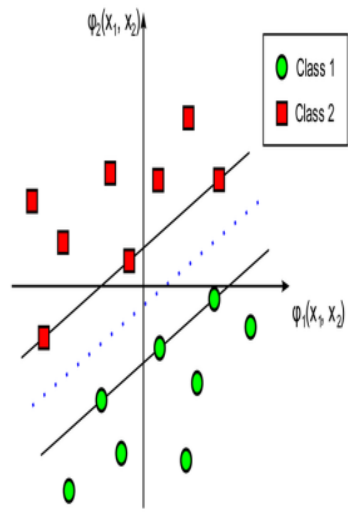

(b)
Fig 2: SVM classification for two classes

\section{EXPERIMENTAL RESULTS}

\section{A.Datasets}

We had collected standard flavia datasets. There are 500 samples of leaf images. Feature values are extracted from standard datasets and they are trained and tested.
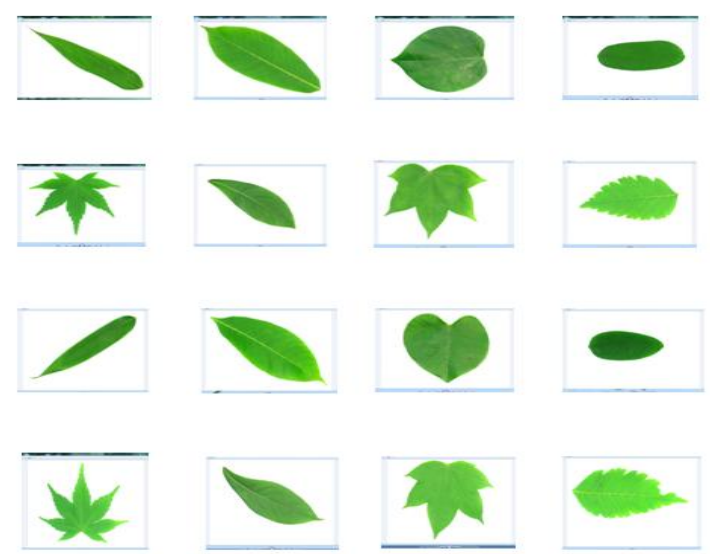

Fig 3:Dataset samples

\section{B. Performance Measure.}

In this session we are discussing about the performance of the proposed system.

Table-I : Percentage of accuracy for different trials

\begin{tabular}{|c|c|c|c|c|c|}
\hline $\begin{array}{c}\text { Trials- } \\
\text { \% }\end{array}$ & $\mathbf{t 1}$ & $\mathbf{t 2}$ & $\mathbf{t 3}$ & $\mathbf{t 4}$ & $\mathbf{t 5}$ \\
\hline $30-40$ & 52 & 44 & 51.7 & 46.5 & 48.5 \\
\hline $40-60$ & 50 & 43.3 & 46.6 & 47.3 & 51 \\
\hline $50-50$ & 51.2 & 51.2 & 52 & 52 & 50.4 \\
\hline
\end{tabular}

\begin{tabular}{|c|c|c|c|c|c|}
\hline $60-40$ & 50.5 & 50 & 50.5 & 51.5 & 48 \\
\hline $70-30$ & 45.3 & 48.6 & 49.3 & 45.3 & 50 \\
\hline $80-20$ & 51 & 54 & 52 & 46 & 49 \\
\hline $90-10$ & 62 & 62 & 50 & 52 & 50 \\
\hline
\end{tabular}

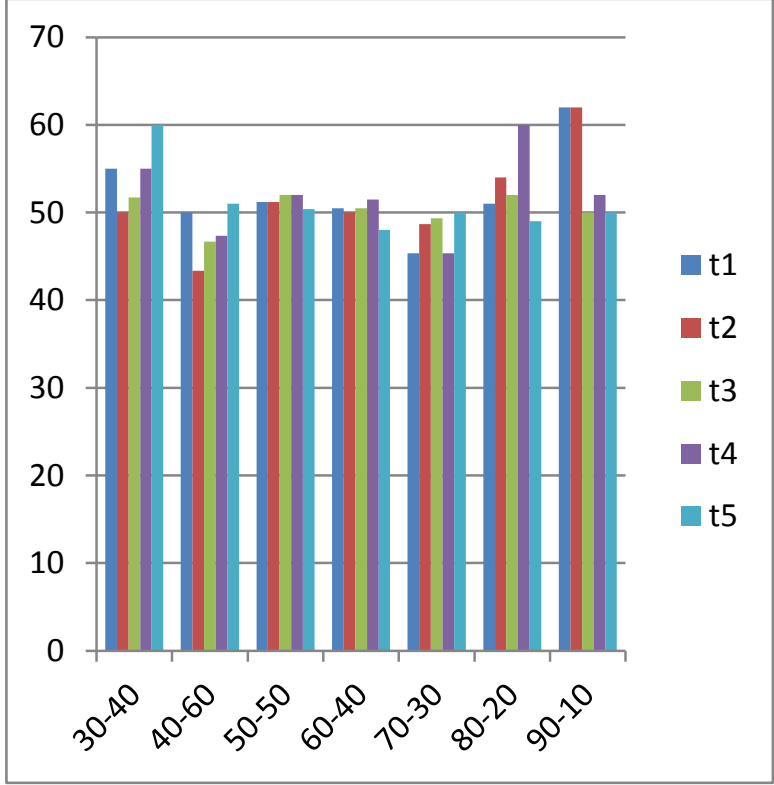

Fig 4: Accuracy of the proposed system

$\mathrm{X}$ axis $\rightarrow$ Trials

$\mathrm{Y}$ axis $\rightarrow$ Percentage of accuracy obtained

\section{CONCLUSIONS}

Here in this Paper, we can easily classify particular leaf using specific methods. The methodologies used in this work will helps in easy recognition of specified species. GLCM matrix easily helps us to extract the texture features. The GLCM statistical properties allows for faster recognition of different varieties of leaves. The classifier used in this work helps in easy classification of leaves with faster and accurate classification.

\section{FUTURE ENHANCEMENT}

Future directions can include enhancing the research methodologies by making it working for several plant species. Here in this project we are considering texture feature. By considering other features like shape, area and other classification methods such as KNN, PCA, ANN, PNN we can better enhance this project. In future we can build a mobile application of the proposed system, using this one can easily and efficiently classify the leaves.

\section{REFERENCES}

[1] M. Z. Rashad, B. S. el-Desouky,and Manal S. Khawasik, "Plants Images Classification Based on Textural Features using Combined Classifier", International Journal of Computer Science \& Information Technology (IJCSIT), Vol 3, No. 4, August 2011,pp.93-100.

[2] Abdul Kadir, Lukito Edi Nugroho, and Paulus Insap Santosa, "Leaf classification using shape, color, and 
texture”, International Journal of Computer Trends \& Technology (IJCTT), July-August 2011,pp.225-230.

[3] C. S. Sumathi and A. V. Senthil Kumar, "Edge and Texture Fusion for Plant Leaf Classification", International Journal of Computer Science and Telecommunications, Vol 3, Issue 6, June 2012,pp. 6-9.

[4] T. Beghin, J. S. Cope, P. Remagnino, \& S. Barman, "Shape and texture based plant leaf classification", Advanced Concepts for Intelligent Vision Systems (ACVIS),Vol 6475, 2010,pp.45-353.

[5] C. H. Arun, W. R. Sam Emmanuel, and D. Christopher Durairaj, "Texture Feature Extraction for Identification of Medicinal Plants and Comparison of Different
Classifiers", International Journal of Computer Applications (0975-8887), Vol 62,No.12,January 2013, pp.19.

[6] J. S. Cope, P. Remagnino, S. Barman, and P. Wilkin, "Plant texture classification using gabor cooccurrences," In Advances in Visual Computing, Springer Berlin Heidelberg, 2010, pp.669-677.

[7] Shayan Hati BTech Student, "Plant Recognition from Leaf Image through Artificial Neural Network ", Department of Civil Engineering, Indian Institute of Technology (IIT), Kharagpur International Journal of Computer Applications (0975 - 8887) Volume 62No.17, January 2013 